\title{
Anesthetic considerations in robotic mitral valve surgery
}

\author{
Kent H. Rehfeldt, J. Valery Andre, Matthew J. Ritter \\ Department of Anesthesiology, Mayo Clinic, Rochester, MN, USA \\ Correspondence to: Kent H. Rehfeldt, MD, FASE. Department of Anesthesiology, Mayo Clinic, 200 First Street SW, Rochester, MN 55905, USA. \\ Email: rehfeldt.kent@mayo.edu.
}

\begin{abstract}
The robotic approach to cardiac surgery offers patients numerous potential advantages compared with a traditional sternotomy approach including shorter hospital length of stay, reduced pain, fewer blood transfusions, and a quicker return to normal daily activities. At the same time, robotic cardiac surgery requires that the anesthesiologist employs several subspecialty skillsets in order to provide optimal care for these patients. Multiple different regional anesthesia techniques may be used to improve analgesia, reduce opioid dosages, and facilitate rapid extubation at the conclusion of the case. Several peripheral cannulation strategies for cardiopulmonary bypass (CPB) exist and the anesthesia team may assist with percutaneous cannulation of the superior vena cava (SVC) or positioning of an endo-pulmonary vent. Similarly the anesthesiologist may be asked to percutaneously cannulate the coronary sinus for retrograde cardioplegia delivery. The need for one-lung ventilation (OLV) and heavy reliance on transesophageal echocardiography (TEE) occupy much of the anesthesiologist's attention during these cases. Variations in institutional practice exist. Reviews of current practice and future studies may help refine the anesthetic approach to robot-assisted cardiac surgery.
\end{abstract}

Keywords: Anesthesia; cardiac surgery; transesophageal echocardiography (TEE); cardiopulmonary bypass (CPB); robotics

Submitted Jan 06, 2017. Accepted for publication Jan 14, 2017.

doi: $10.21037 /$ acs.2017.01.10

View this article at: http://dx.doi.org/10.21037/acs.2017.01.10

The practice of robot-assisted mitral valve repair (MVR) offers numerous potential benefits to patients including shorter hospital length of stay (1-3), reduced postoperative pain (4), a lower rate of surgical site infection (4), diminished incidence of blood product transfusion $(5,6)$, a faster return to normal daily activities $(5,7)$, and greater overall patient satisfaction (4). Anesthesiologists play in a key role in facilitating optimal outcomes after robotic MVR. However, just as robotic and minimally-invasive approaches offer unique opportunities for improved patient outcomes, they also pose specific challenges to the anesthesia team. The successful perioperative care of patients undergoing robot-assisted MVR requires that the anesthesiologist employ numerous subspecialty skillsets including regional anesthesia and analgesia techniques, elements of thoracic anesthesia practice, in particular one-lung ventilation (OLV), cardiac anesthesia, and transesophageal echocardiography (TEE) (8). One study noted an increased requirement for anesthesia personnel for the completion of robotic cardiac surgery compared with traditional approaches (9).

\section{Preoperative planning}

Optimal patient outcome following cardiac surgery requires deliberate communication and planning between surgeons, anesthesiologists, perfusionists, nurses, and surgical assistants. The need for planning and preoperative discussion is especially critical prior to robotic cardiac surgical procedures since surgical and anesthetic techniques often differ from the "standard" approach and numerous possible options exist for accomplishing similar goals. For example, the site and timing of regional anesthesia and analgesia interventions must be clarified. The conduct of cardiopulmonary bypass (CPB) must be planned, in particular the number, type, and location of venous 
cannulae. Similarly, the means by which cardioplegia will be administered should be delineated. Formulation of a preliminary plan for immediate postoperative care ensures that appropriate personnel and bed space are available. For example, if extubation in the operating room is planned, the patient may be transferred directly to a step-down unit or recovery room, rather than an intensive care setting.

\section{Analgesia}

While some investigators have noted reduced postoperative pain and decreased requirements for analgesic medications following robotic cardiac surgery when compared with the standard sternotomy (5), robot-assisted MVR is not painfree. Some reports have noted similar postoperative pain scores whether cardiac surgery is performed by standard sternotomy or using a minimally-invasive approach (10). Certainly, an opioid-based, intravenous analgesia regimen may be used for either standard sternotomy or minimallyinvasive approaches for mitral valve operations. However, opioid-based analgesia regimens are accompanied by notable side effects, in particular postoperative nausea and vomiting and delayed emergence from anesthesia. The latter may confound attempts to extubate the patient promptly at the conclusion of surgery while the former contributes significantly to decreased patient satisfaction.

Because the typical conduct of robot-assisted MVR involves several small incisions in the right hemithorax, a number of regional anesthesia and analgesic techniques may be employed. Regional techniques that may be selected include the administration of intrathecal opioids or local anesthetics, thoracic epidural catheter placement, intercostal nerve blocks (ICNB), or paravertebral nerve blocks (PVNB) (1). The local infiltration of long-acting, liposomal bupivacaine (Exparel, Pacira, Parsippany, NJ, USA) into the incisions represents another alternative. Recently a team of investigators noted improved postoperative pain scores in patients who received liposomal bupivacaine infiltration compared with standard bupivacaine at the conclusion of cardiac surgery (11).

Some anesthesiologists have used intrathecal opioids with good success in patients undergoing cardiac surgical procedures and we have used this technique on occasion. Our approach to intrathecal analgesia involves the preinduction injection of $300 \mathrm{mcg}$ of preservative-free morphine administered as a single shot in the lumbar intrathecal space using a 25 gauge needle combined with a long-acting local anesthetic injected into the incisions at

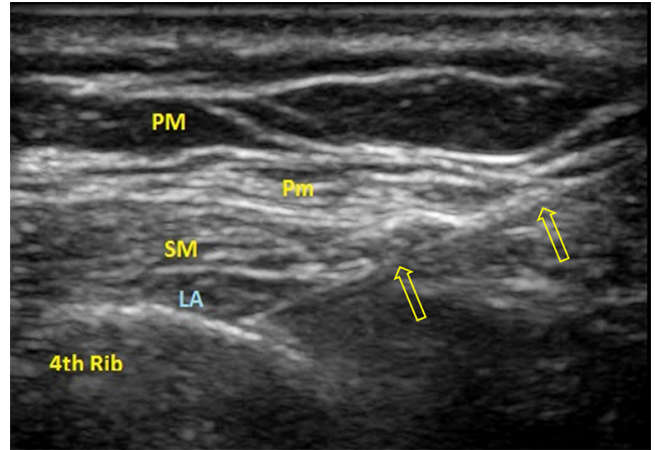

Figure 1 Ultrasound-guided Pecs II block. The needle (arrows) is visualized depositing local anesthetic (LA) between the serratus anterior muscle (SM) and the $4^{\text {th }}$ rib. The pectoralis major (PM) and pectoralis minor $(\mathrm{Pm})$ muscles are also labeled.

the end of the case by the surgeons. Since no paravertebral block is performed, the surgeon can inject a larger volume of either plain bupivacaine or liposomal bupivacaine at the end of the operation. In our experience, this allows for extubation at the end of the case and excellent analgesia for 12-24 hours after surgery. However, this still requires several minutes to perform the intrathecal injection prior to the start of the procedure and many anesthesiologists are reluctant to utilize neuraxial techniques prior to administration of large doses of heparin.

More recently, we have begun to utilize the newer regional anesthetic blocks described by Blanco and colleagues, including the pectoralis blocks (Pecs I and Pecs II) $(12,13)$ and serratus plane block (14). These blocks are simple to perform with ultrasound guidance, they can be performed after the patient is asleep, and there is no risk for central spread with resulting sympathectomy. The Pecs II block (Figure 1) reliably blocks T2-T4 dermatomes, with variable coverage to T6 (13). Whereas, the serratus plane block reliably covers T2-T7, with variable spread to T9 dermatomes (14). Thus, a single injection can cover multiple port sites, primary incision and chest tube site. Although there is no literature yet on the use of these blocks for minimally invasive cardiac surgery, the Pecs blocks are effective for mastectomy pain $(15,16)$ and the serratus plane block has been used successfully to treat thoracotomy pain (17) and discomfort from multiple rib fractures (18).

Although we have utilized intrathecal analgesia, pectoralis blocks and serratus plane blocks, the vast majority of patients undergoing robot-assisted MVR at our institution have received pre-induction PVNB. 
These blocks are relatively easy to perform, particularly with ultrasound guidance. The patient assumes a sitting position on the side of the operating room bed with his or her arms and head supported by pillows that have been placed on a bedside stand. After initiation of supplemental oxygen and intravenous sedation, a series of three, rightsided paravertebral injections are performed. Advantages of PVNB include spread of local anesthetic over multiple dermatomes including the main, "working" incision as well as other smaller port incisions for instrument placement. Compared with placement of a thoracic epidural catheter, PVNB are associated with a reduced risk of neuraxial hematoma in a heparinized patient as well as a lower risk of hypotension from sympathetic blockade. The inclusion of PVNB into our anesthetic management plan along with the associated decrease in the use of systemic opioid medications has allowed us to extubate almost all patients in the operating room at the conclusion of surgery (19).

\section{OLV and capnothorax}

Since robot-assisted MVR is accomplished via small incisions and working ports in the right chest, the procedure requires collapse of the right lung, insufflation of carbon dioxide $\left(\mathrm{CO}_{2}\right)$ into the right chest, and isolated ventilation of the left lung. Lung isolation may be accomplished by means of either a double-lumen endotracheal tube (DLETT) or the use of a standard, single-lumen endotracheal tube (SLETT) along with a bronchial blocker. In our practice, we have used both methods, although we favor the use of a left-sided DLETT when feasible. Although initial placement of a DLETT is more challenging, we find it easier to deflate the right lung when compared with SLETT with a bronchial blocker. Because of the shorter distance between the carina and the upper lobe bronchus on the right, it may be difficult to prevent a right-sided bronchial blocker from occluding air flow from the right upper lobe. In the event that the right upper lobe bronchus is even partially occluded by the bronchial blocker, deflation of this segment becomes difficult. We have also found that dislodgment of the right-sided bronchial blocker occurs relatively frequently with repetitive inflation and deflation and the right lung, particularly following CPB. Finally, the application of continuous positive airway pressure (CPAP) to the right lung is more difficult when a bronchial blocker is in place. Nonetheless, placement of a SLETT with a bronchial blocker may be preferred if the patient proves difficult to intubate, precluding the use of a DLETT.
Some authors advocate changing a DLETT to a SLETT at the conclusion of robotic cardiac surgery (5). In our own practice this is almost never necessary for two reasons. First, nearly every patient is extubated in the operating room at the conclusion of surgery, a process that is aided tremendously by the use of regional anesthesia with minimization of systemic opioid administration. Second, in the rare instance in which ventilation is to be maintained in the ICU, our critical care physicians are comfortable caring for patients who are ventilated with a DLETT. The duration of mechanical ventilation in the ICU following robotic MVR is seldom more than an hour or two and the availability of smaller suction catheters facilitates pulmonary toilet.

Intraoperative hypoxemia associated with OLV during robotic cardiac surgery is well-documented $(1,5,8)$. Although it would be helpful identify those patients in whom OLV-associated hypoxemia was most likely to develop, we are unaware of any studies indicating the predictive value of preoperative pulmonary testing in this patient population. Therefore, we do not routinely perform preoperative pulmonary testing prior to robotic cardiac surgery. Hypoxemia tends to be particularly problematic following $\mathrm{CPB}$, even if OLV poses no problem prior to $\mathrm{CPB}$. Some investigators have noted a reduction in the $\mathrm{PaO}_{2} / \mathrm{FiO}_{2}$ ratio by $50 \%$ following $\mathrm{CPB}$ when compared with pre-CPB values in patients undergoing minimally invasive cardiac operations (9). Increased shunting of blood through the non-ventilated lung and impaired ventilationperfusion matching in the ventilated lung have been proposed as mechanisms for the hypoxemia noted in these cases (9). The treatment of hypoxemia during OLV may include usual strategies such as the application of positive end-expiratory pressure (PEEP) to the ventilated lung and the application of CPAP to the non-ventilated lung. The use of PEEP in the ventilated lung is quick and easy to implement but may promote the shunting of blood to the non-ventilated lung and paradoxically worsen oxygenation. Providing CPAP to the non-ventilated lung at level of 5 to $10 \mathrm{cmH}_{2} \mathrm{O}$ reliably improves oxygenation. However, it has been our observation that the application of even low levels of CPAP to the non-ventilated right lung is quickly detected by the surgeon who is viewing the field with magnified, stereoscopic vision. In general, our surgeons much prefer intermittent two-lung ventilation rather than the application of CPAP to the right lung. Therefore $\mathrm{OLV}$-associated hypoxemia is usually managed by a brief interruption in the intrathoracic portion of the operation 
during which time oxygen saturation is restored by means of two-lung ventilation. During these periods, the surgeon attends to other aspects of the operation, such as closure of groin incisions.

The insufflation of $\mathrm{CO}_{2}$ into the right chest is commonly performed following deflation of the right lung. Insufflation of $\mathrm{CO}_{2}$ into the chest reduces the amount of intracardiac air present at the conclusion of $\mathrm{CPB}$ and diminishes the likelihood of a surgical site fire. Hemodynamic compromise resulting from a tension capnothorax is possible though we have encountered this complication only several times in nearly 700 robotic mitral operations. If pressure in the right hemithorax does not exceed $10 \mathrm{mmHg}$ and rates of insufflation are held below 2 to $3 \mathrm{~L} / \mathrm{min}$, the risk of a tension capnothorax is reduced $(5,20)$.

One unique concern related to robotic MVR involves defibrillation. The small incision does not permit the performance of internal defibrillation, necessitating the use of external pads. Modification of external pad position is needed because of the extent of the surgical field. Typically we place one pad on the back, to the left of the spine. The second pad is near the apex of the heart at the left anterior axillary line. The presence of a capnothorax further complicates the situation, since $\mathrm{CO}_{2}$ acts as an electrical insulator, further hampering defibrillation efforts. If initial external defibrillation efforts prove unsuccessful, consideration should be given to resuming two-lung ventilation to reduce electrical impedance through the chest $(1,5,8,21)$. Fortunately, we rarely encounter patients who are resistant to more than three or four defibrillation attempts when using the aforementioned pad position together with a biphasic defibrillator and energy levels of 120 to 200 Joules. Intravenous amiodarone is typically given if one or two defibrillation attempts are unsuccessful. Placement of epicardial pacing wire is difficult during robotic mitral surgery and we rarely place epicardial or transvenous pacing wires in these cases. In uncommon cases where pacing is desired ( $<1 \%$ of robotic surgeries), a single, bipolar, right ventricular epicardial lead can be used.

\section{Line placement and bypass cannulation}

The notion of robotic MVR implies peripheral cannulation for CPB. Nonetheless different CPB cannulation strategies may be employed, particularly for the venous return lines. Similarly, different strategies for cardioplegia delivery may be selected based on surgeon preference or patient characteristics. Anesthesiologists may be tasked with placing

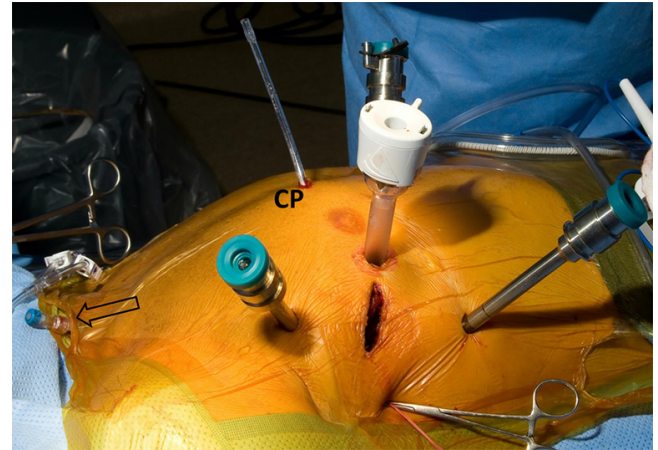

Figure 2 Surgical site photograph taken from the right side of the bed. A capped, 5 French cannula (arrow) has been placed in the right internal jugular (RIJ) vein and prepped into the field. This will be re-wired to a superior vena cava (SVC) cannula. A cannula for venting and antegrade cardioplegia delivery into the ascending aorta is also indicated (CP).

lines that will be used for venous drainage or cardioplegia administration. The location and number of such lines may, in turn, influence the selection of additional lines used for pressure monitoring, fluid administration, or drug delivery.

The primary method of venous drainage for CPB is placement of a cannula via the femoral vein that is typically advanced into the right atrium (RA) or superior vena cava (SVC). In addition, supplementary venous drainage may be accomplished by means of a 15 to 18 French cannula introduced into the right internal jugular (RIJ) vein and advanced into the SVC under TEE guidance. This SVC cannula may be placed entirely by the anesthesiologist with tubing passed around the surgical drapes to the $\mathrm{CPB}$ machine. Alternatively, as is our practice, a 5 French singlelumen cannula is inserted by the anesthesiologist into the RIJ, close to the clavicle (Figure 2). This line is flushed, capped and prepped into the surgical field. Later, under TEE guidance, the surgeon uses this 5 French cannula to introduce a guidewire, followed by dilators, and finally the wire-reinforced venous cannula. Supplementary venous drainage may also be provided by a commercially-available endo-pulmonary vent. This balloon tipped catheter has a design similar to a short pulmonary artery catheter and is inserted into the RIJ by mean of an introducer sheath. The tip of the 9 French, endo-pulmonary vent is advanced with TEE guidance to a position in the main pulmonary artery, within one to two centimeters of the pulmonary artery bifurcation and then connected by vacuum-assisted drainage into the $\mathrm{CPB}$ circuit. After using the endo-pulmonary vent 
for an initial series of robotic MVR cases, we have since abandoned it in favor of a 15 to 18 French SVC cannula introduced via the RIJ. It is our clinical impression that the latter system provides better venous drainage at a lower cost than the endo-pulmonary vent.

In addition to the 5 French introducer that is prepped into the surgical field, we typically place a second line into the RIJ at a position that allows placement of surgical drapes between the two. Typically an 8.5 French, quad-lumen central venous catheter is selected as the second RIJ line, although a 9 French introducer may be chosen if peripheral venous access is limited. We do not use a pulmonary artery catheter for robotic MVR cases. If an endo-pulmonary vent is used, pulmonary artery pressures can be transduced prior to $\mathrm{CPB}$. Once protamine is given, the endo-pulmonary vent is generally removed.

The RIJ may also be used to place a percutaneous coronary sinus catheter for retrograde cardioplegia delivery. Retrograde cardioplegia may be chosen as the sole method of cardiac arrest by choice or because of patient factors (significant aortic regurgitation). We have used a percutaneous coronary sinus catheter for retrograde cardioplegia delivery in a limited number of cases. In these cases we used a both TEE and fluoroscopy with contrast administration to confirm correct catheter position in the coronary sinus. During placement of percutaneous coronary sinus catheter, we have found the modified TEE bicaval view at approximately $110^{\circ}$ to be particularly helpful. If the catheter moves toward the tricuspid valve or right atrial appendage, it is rotated counterclockwise and reinserted. Should the catheter be directed toward the inferior vena cava, it is rotated clockwise prior to additional attempts.

Arterial pressure monitoring is accomplished by means of a left radial artery catheter. The left radial artery is preferentially chosen because the right arm is positioned along the patient's right side in front of the bedside surgeon. Should a right radial arterial line become damped or otherwise nonfunctional during the operation, it would be considerably more difficult to troubleshoot. However, if endo-aortic balloon occlusion of the ascending aorta with antegrade cardioplegia is planned, then bilateral radial arterial catheters are placed. Under these circumstances, damping of the right radial arterial pressure waveform is presumed to represent migration of the endoaortic balloon with innominate artery occlusion. Intraoperative cerebral oximetry has also been suggested as a means to detect endo-aortic balloon migration (5). A decline in right-sided cerebral oximeter readings should prompt
TEE confirmation of aortic balloon position. Our own surgical practice has never included the endo-aortic balloon clamping and cardioplegia delivery system. Instead, our surgeons favor a long transthoracic, or Chitwood, clamp together with antegrade cardioplegia that is administered by a cannula placed into the ascending aorta via a small port incision located to the right of the sternum (Figure 2). Blood-based crystalloid cardioplegia is given. We have infrequently used retrograde cardioplegia administered via a percutaneously-placed coronary sinus catheter.

\section{TEE}

The practice of robot-assisted MVR depends heavily on intraoperative TEE. In addition to precise delineation of mitral pathology and evaluation of the surgical repair, TEE is used to detect additional findings that may impact the conduct of the operation. The presence of more than mild aortic regurgitation may necessitate the administration of retrograde cardioplegia. Atrial level shunts may complicate passage of femoral venous guidewires and cannulae.

However, the need for real-time guidance during placement of guidewires and cannulae represents a unique role for TEE during robotic and minimally-invasive cardiac surgery. During cannulation of the femoral artery, continuous TEE imaging ensures that guidewire passage into the descending aorta has been successful. If the endoaortic balloon occlusion system is planned, TEE is further used to verify final balloon position approximately $2 \mathrm{~cm}$ above the aortic root (8). If the endo-aortic balloon catheter is not utilized, the aortic cannula cannot be visualized with TEE. Passage of a guidewire from the femoral vein is also monitored with TEE. Ideally the guidewire will pass through the RA and with the tip residing in the SVC. We prefer to have the femoral venous cannula tip positioned in the SVC with its tip approximately 4 to $5 \mathrm{~cm}$ from the RA-SVC junction. When thus positioned, the chances that this cannula will be dislodged during the manipulation of the atrial retractor are decreased. Occasionally the femoral venous guidewire is malpositioned across a patent foramen ovale or becomes coiled in the RA appendage. The position of the guidewire and venous cannula introduced into the SVC from the RIJ are also monitored. Occasionally the tip of this SVC cannula overlaps the tip of the cannula introduced via the femoral vein, though this does not seem to be problematic.

If percutaneous coronary sinus cannulation and placement of an endo-pulmonary vent are planned, TEE is 

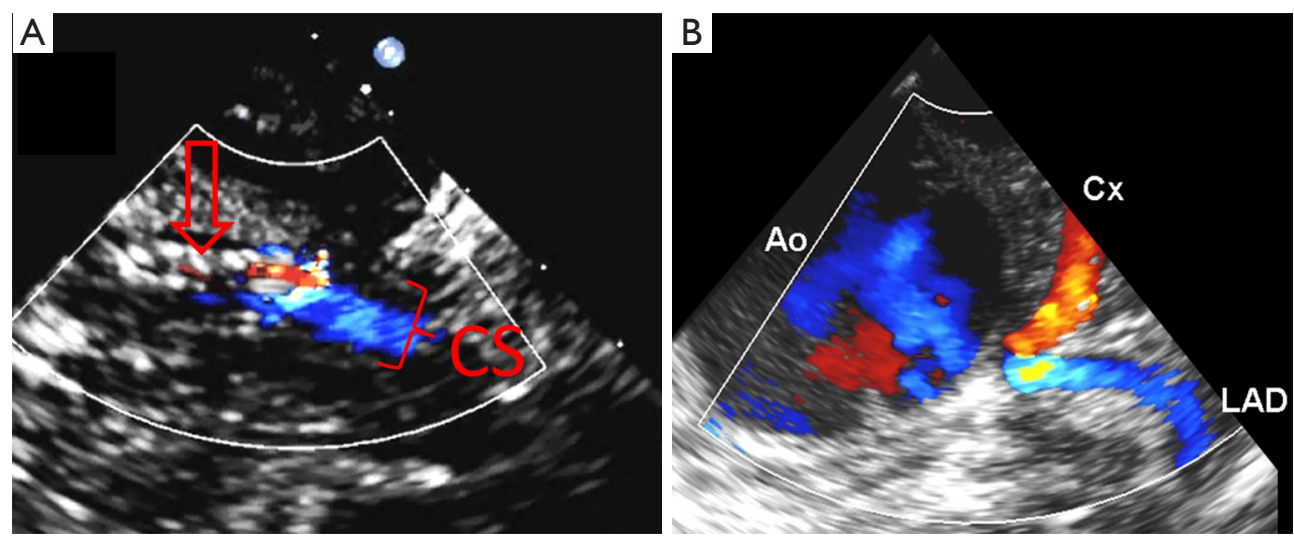

Figure 3 TEE imaging of retrograde (A) and an antegrade (B) cardioplegia delivery. (A) Transesophageal echocardiographic image of the coronary sinus (CS) demonstrating the administration of retrograde cardioplegia via a percutaneously-introduced catheter (arrow); (B) transesophageal echocardiographic image of the aortic root (Ao) demonstrating flow of antegrade cardioplegia into the left circumflex (Cx) and left anterior descending (LAD) coronary arteries.

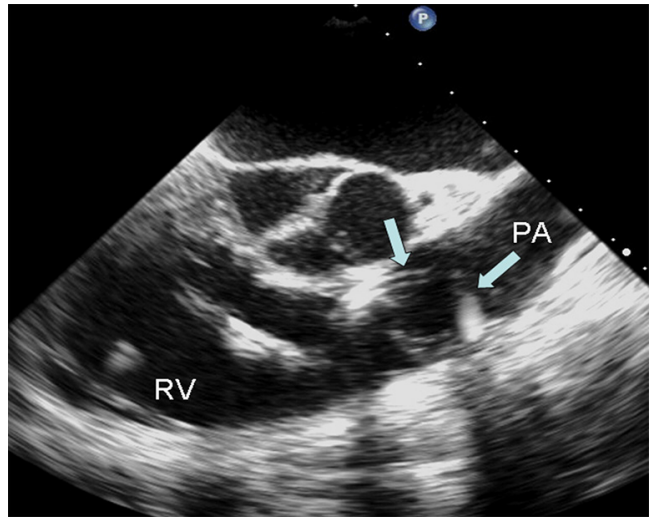

Figure 4 Transesophageal echocardiographic image demonstrating looping of an endo-pulmonary vent in the right ventricular (RV) outflow tract and proximal pulmonary artery (PA).

also invaluable in confirming the positions of these devices as well. Both two-dimensional and three-dimensional TEE imaging may be used to visualize proper cannulation of the coronary sinus. The addition of color Doppler can verify flow of cardioplegia into the coronary sinus during $\mathrm{CPB}$ (Figure $3 A$ ). The delivery of antegrade cardioplegia is also routinely imaged in our practice (Figure $3 B$ ). Although an electrically silent ECG is reassuring, we prefer to visualize the flow of cardioplegia into the left main and right coronary arteries. The use of TEE during the initial administration of cardioplegia also excludes the presence of significant aortic regurgitation and left ventricular distention. Confirmation of proper location of an endopulmonary vent in the main pulmonary artery necessitates
TEE imaging as well. On occasion, we have observed coiling of the endo-pulmonary vent in the PA or right ventricular outflow tract (Figure 4), complications that would potentially go undetected without TEE visualization.

Once the left atriotomy is repaired, the patient is weaned from CPB. At this time, continuous TEE imaging is used to monitor air evacuation via the cardioplegia/vent line that was placed percutaneously into the ascending aorta. The results of the mitral repair are also assessed. After air has been evacuated from the left heart chambers and the mitral repair deemed satisfactory, CPB is briefly resumed. During this second, brief period of CPB, the cardioplegia/vent line is removed from the aorta under transient, low-flow conditions. Subsequently, the patient is weaned again from $\mathrm{CPB}$ and a complete TEE examination is repeated.

\section{Conclusions}

The robotic approach has been demonstrated to offer significant benefits to patients in need of mitral surgery. The unique aspects of this practice create both complexities and opportunities for the anesthesiologists who participate in these operations. While numerous options exist for the perioperative management of these cases, we anticipate that growing clinical experience and future investigations will further refine our care of these patients.

\section{Acknowledgements}

None. 


\section{Footnote}

Conflicts of Interest: The authors have no conflicts of interest to declare.

\section{References}

1. Rehfeldt KH, Mauermann WJ, Burkhart HM, et al. Robot-assisted mitral valve repair. J Cardiothorac Vasc Anesth 2011;25:721-30.

2. Chitwood WR Jr, Rodriguez E, Chu MW, et al. Robotic mitral valve repairs in 300 patients: a single-center experience. J Thorac Cardiovasc Surg 2008;136:436-41.

3. Deeba S, Aggarwal R, Sains P, et al. Cardiac robotics: a review and St. Mary's experience. Int J Med Robot 2006;2:16-20.

4. Woo YJ. Robotic cardiac surgery. Int J Med Robot 2006;2:225-32.

5. Bernstein WK, Walker A. Anesthetic issues for robotic cardiac surgery. Ann Card Anaesth 2015;18:58-68.

6. Woo YJ, Nacke EA. Robotic minimally invasive mitral valve reconstruction yields less blood product transfusion and shorter length of stay. Surgery 2006;140:263-7.

7. Bonaros N, Schachner T, Wiedemann D, et al. Quality of life improvement after robotically assisted coronary artery bypass grafting. Cardiology 2009;114:59-66.

8. Wang G, Gao C. Robotic cadiac surgery: an anesthetic challenge. Postgrad Med J 2014;90:467-74.

9. Kottenberg-Assenmacher E, Kamler M, Peters J. Minimally invasive endoscopic Port-Access intracardiac surgery with one-lung ventilation: Impact on gas exchange and anaesthesia resources. Anaesthesia 2007;62:231-8.

10. Cogan J, Lebon J, Rochon A, et al. Pain levels after minimally invasive cardiac surgery: Results for the first 7 days. Anesth Analg 2010;110:1-101.

11. Balkhy HH, Arnsdorf S, Krienbring D, et al. Liposomal bupivacaine for postsurgical analgesia in patients

Cite this article as: Rehfeldt KH, Andre JV, Ritter MJ. Anesthetic considerations in robotic mitral valve surgery. Ann Cardiothorac Surg 2017;6(1):47-53. doi: 10.21037/ acs.2017.01.10 undergoing robotically assisted cardiac surgery.

Innovations (Phila) 2015;10:416-9.

12. Blanco R. The 'pecs block": a novel technique for providing analgesia after breast surgery. Anaesthesia 2011;66:847-8

13. Blanco R, Fajardo M, Parras Maldonado T. Ultrasound description of Pecs II (modified Pecs I): a novel approach to breast surgery. Rev Esp Anestesiol Reanim 2012;59:470-5.

14. Blanco R, Parras T, McDonnell JG, et al. Serratus plane block: a novel ultrasound-guided thoracic wall nerve block. Anaesthesia 2013;68:1107-13.

15. Kulhari S, Bharti N, Bala I, et al. Efficacy of pectoral nerve block versus thoracic paravertebral block for postoperative analgesia after radical mastectomy: a randomized controlled trial. Br J Anaesth 2016;117:382-6.

16. Hetta DF, Rezk KM. Pectoralis-serratus interfascial plane block vs thoracic paravertebral block for unilateral radical mastectomy with axillary evacuation. J Clin Anesth 2016;34:91-7.

17. Okmen K, Okmen BM, Uysal S. Serratus anterior plane (SAP) block used for thoracotomy analgesia: A case report. Korean J Pain 2016;29:189-92.

18. Kunhabdulla NP, Agarwal A, Gaur A, et al. Serratus anterior plane block for multiple rib fractures. Pain Physician 2014;17:E651-3.

19. Lynch JJ, Mauermann WJ, Pulido JN, et al. Use of paravertebral blockade to facilitate early extubation after minimally invasive cardiac surgery. Semin Cardiothorac Vasc Anesth 2010;14:47-8.

20. Chauhan S, Sukesan S. Anesthesia for robotic cardiac surgery: an amalgam of technology and skill. Ann Card Anaesth 2010;13:169-75.

21. Hatton KW, Kilinski LC, Ramaiah C, et al. Multiple failed external defibrillation attempts during robot-assisted internal mammary harvest for myocardial revascularization. Anesth Analg 2006;103:1113-4. 